\title{
Flying-spot lock-in thermography and its application to thickness measurement and crack detection
}

by U. Netzelmann

Fraunhofer Institute for Nondestructive Testing IZFP, Campus E3 1, 66123 Saarbrücken, Germany, udo.netzelmann@izfp.fraunhofer.de

\begin{abstract}
A heating laser beam was scanned periodically along a testing path on the surface of a test object. The thermal response of the beam was recorded by an infrared camera. Using a lock-in thermography algorithm, amplitude and phase images were generated. The phase image is corrected for effects due to the beam movement. A first application shows the contact-free determination of the steel sheet thickness at forming edges. Calibration of phase values to thickness was achieved by using an analytical model of thermal wave transmission. A second application is the detection of a perpendicular surface crack in steel.
\end{abstract}

\section{Introduction}

Thermographic techniques using a laser beam that is moved with constant speed on a test object are attractive as they allow to find perpendicular surface cracks which would otherwise not be detected. This has been studied in detail in work related to flying-spot thermography [1-6]. A common difficulty with this technique is the superposition of multiplicative disturbing influences. These can be emissivity contrasts from contaminations, corrosion or scratches. Other disturbances are due to spatially varying power density of the excitation laser radiation at curved surface regions. In the past, approaches were described to recognize some of these disturbances and to suppress them [7-8]. When using an infrared camera as detector, gradient formation and a special processing of the image sequences led to an improvement of the image contrasts [9].

The efforts to suppress emissivity variations and other disturbances are due to the fact, that e. g. the thermal contrast due to surface cracks is usually small compared to the contrast of the sound material. This also holds true, when the thermal contrast to be detected results from heat flow components perpendicular to the surface, if thickness variations or bonding defects have to be detected.

For broad illumination by lamps, lock-in techniques and pulse-phase analysis are well established. For point-like excitation, intensity modulated laser beams and lock-in detection are used in thermal wave microscopy [10] or in photothermal thickness measurement devices [11]. Phase signals and phase images are generated, which exhibit the desired suppression of disturbances. Even if the laser is rastering the surface step-wise or continuously slowly moving, the signals are interpreted as resulting from a static measurement. Errors occurring due to the relative movement between heating source and test object are treated as a small disturbance [12-15].

In spite of significant previous efforts to suppress disturbance in flying-spot techniques, it is desirable to find new approaches for improving the signal quality.

\section{Principle of measurement}
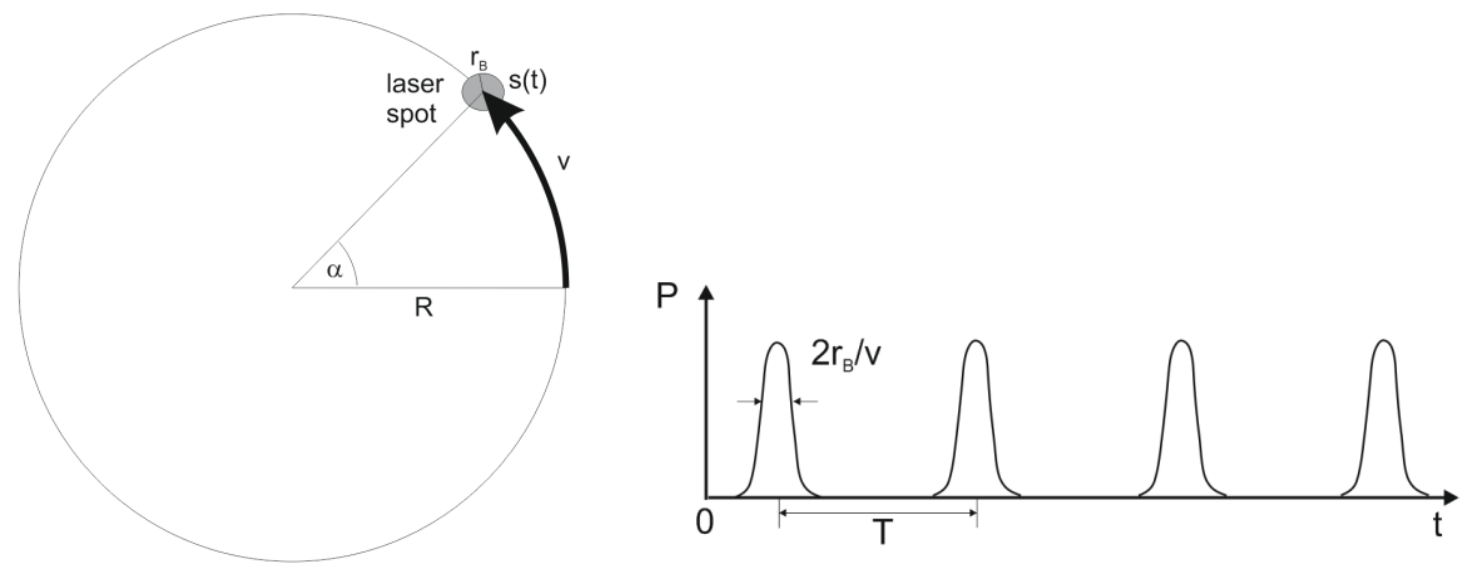

Fig. 1. Sketch of the movement of a laser spot (left) and the resulting time dependent excitation at a fixed position on the path (right) 
For illustration of the principle it is assumed, that a laser beam with beam diameter $r_{B}$ continuously moves along a circular (without limiting the generality) path with a radius $R$ (Fig. 1, left). If the laser exhibits a Gaussian intensity profile

$$
P=P_{0} e^{-\left(\frac{\Delta r}{2 r_{B}}\right)^{2}}
$$

and if $\mathrm{r}_{\mathrm{B}} \ll R$, a fixed point on the path is exposed to temporally periodic heating pulses, as shown in Fig. 1 , right. These exhibit a temporal width of $2 r_{B} / v$ related to a height of $e^{-0.5}$ or about $61 \%$ of the maximum intensity and are repeated with a period $T$, according to a modulations frequency $f_{M}$, where

$$
T=\frac{2 \pi R}{v} \quad f_{M}=\frac{v}{2 \pi R} .
$$

The laser beam will pass the path many times, for an appropriate application of lock-in processing there should be at least 10 passes. Using an infrared camera, the radiation from the area containing the path is recorded over the whole process. A camera with snapshot-detector should be used. It should have a sufficient frame rate $f_{K}$ in order to allow full recording of the movement without gaps. A criterion for the camera frame rate is

$$
f_{K} \geq \frac{v}{r_{B}}
$$

For larger defect depth or material thickness, a lower frame rate will be sufficient.

Ideally, the start of the camera recording is synchronized with the start of the laser movement. Furthermore, the whole image recording must comprise an integer number of passes. It is useful to run a few passes before the recording in order to ensure periodic heating conditions.

The recorded image sequence will then be processed by a standard lock-in analysis algorithm at the frequency $f_{M}$, resulting in an amplitude and a phase image. Only around the laser path the amplitude image will show a non-zero signal. The phase image exhibits a particularity: If one assumes that zero of time is where the laser beam is at $\alpha=0$ (Fig. 1 , left), there is a spatially dependent geometric background offset of the phase. For a circular path with counterclockwise beam movement, the phase is:

$$
\Phi_{+}=\Phi_{\text {therm }}-\alpha
$$

Here, $\Phi_{\text {therm }}$ is the phase shift of the heat flow. As for a periodic path $\alpha$ varies from 0 to $2 \pi$, and the typical phase shift of the heat flow is usually much smaller, small local effects in the phase image cannot be recognized immediately in the image. In order to solve this problem, there may be two solutions. First, the geometrical phase offset may corrected by an image processing technique. Here, a second approach was selected: The measurement is repeated twice, one with beam movement counter-clockwise and one with beam movement clockwise. For clockwise movement the phase is

$$
\Phi_{-}=\Phi_{\text {therm }}+\alpha
$$

The phase images of both measurements are then averaged numerically, considering the periodicity of the phase with $2 \pi$. As a result, a purely thermal phase image will be generated:

$$
\Phi=\frac{1}{2}\left(\Phi_{+}+\Phi_{-}\right)=\Phi_{\text {therm }}
$$

The situation is basically similar, when a more complicated path is run at constant speed. In a following chapter, some of the approximations related to this proceeding will be discussed.

\section{Experimental}

For excitation, a diode laser with $250 \mathrm{~W}$ maximum power output at a wavelength of $978 \mathrm{~nm}$ is used (Fig. 2). The light energy is coupled to a xy-scanner, that allows to cover a working area of $250 \mathrm{~mm} \times 250 \mathrm{~mm}$. The spot diameter in the focus is $4 \mathrm{~mm}$. A software is available that can be programmed to move the laser spot within the working area along arbitrary paths at controllable speed. A pilot laser allows one to see and check the testing path on the component. 


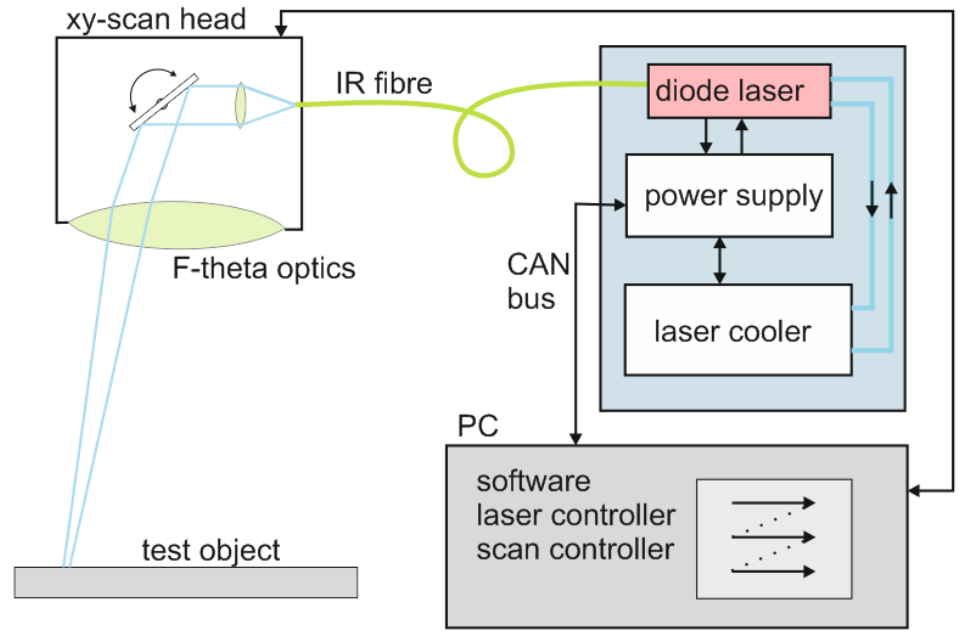

Fig. 2. Scheme of the laser-scan system

A dual-band IR-camera operated in the mid-wave $(4.4-5.2 \mu \mathrm{m})$ was employed. The frame rate was 145 frames/s. Image recording of the camera could be started synchronized with the laser spot movement. The camera was positioned in a way that the scanning area was fully covered in its field-of-view.

\section{Application and results}

When forming components from metal sheets made out of high stiffness steel, due to variations among lots wall thinning at edges may occur at high deformation degrees. In more serious cases this is followed by cracking. The wall thinning is difficult to find visually. Aim of a study was to find out if the lock-in flying spot approach would be able to detect such sheet thickness thinning at the formed edges. Cup-shaped samples with dimensions of about $220 \mathrm{~mm} \times 110 \mathrm{~mm} \times$ $55 \mathrm{~mm}$ with a nominal wall thickness of $0.75 \mathrm{~mm}$ were available (Fig. 3). Using certain process parameters when manufacturing the cups, wall thinning at the edges was provoked for test purposes.

As infrared emission of the sheet may change locally and angular dependent heating power density and thermal emission are present at the curved edges, the emitted radiation alone is no good measure for the sheet thickness. Moreover, there is external radiation which is reflected at the sheet surface. Therefore the lock-in technique described above appears to be a promising testing approach.

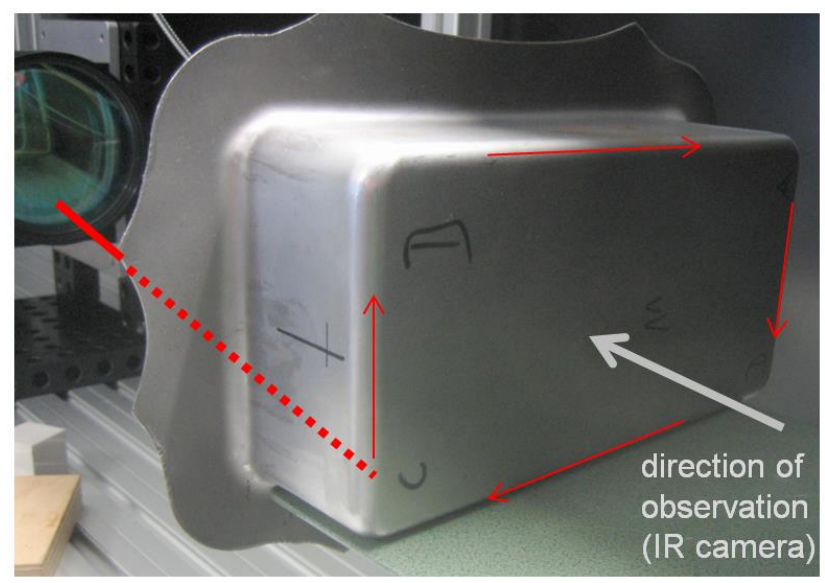

Fig. 3. Photo of a sample in the test setup. Excitation is performed from the rear side by rapid scanning of a laser spot along the inner side of the cup. On the left side, the lens of the scanning head is visible. The red arrows indicate the laser spot movement. Detection by IR camera is performed from the bottom side of the cup (thermal transmission)

Only the rounded edges between the cup bottom and the cup side walls were of interest. In order to detect wall thickness changes, the modulation frequency has to be adapted to the propagation time of the thermal wave through the 
sheet thickness. Due to geometrical restrictions, a thermal transmission measurement was performed. The laser power was $50 \mathrm{~W}$. The edge of the cup bottom was scanned periodically at a speed of about $1000 \mathrm{~mm} / \mathrm{s}$, recording 22 periods with 2048 camera frames. This corresponds to a modulation frequency of $f_{M}=1.56 \mathrm{~Hz}$, at which the thermal diffusion length is adapted to the nominal sheet thickness in order to achieve maximum phase contrast.

The image sequence was then processed by a conventional lock-in FFT algorithm, resulting in an amplitude and a phase image. A result with counter-clockwise laser spot movement is shown in Fig. 4.

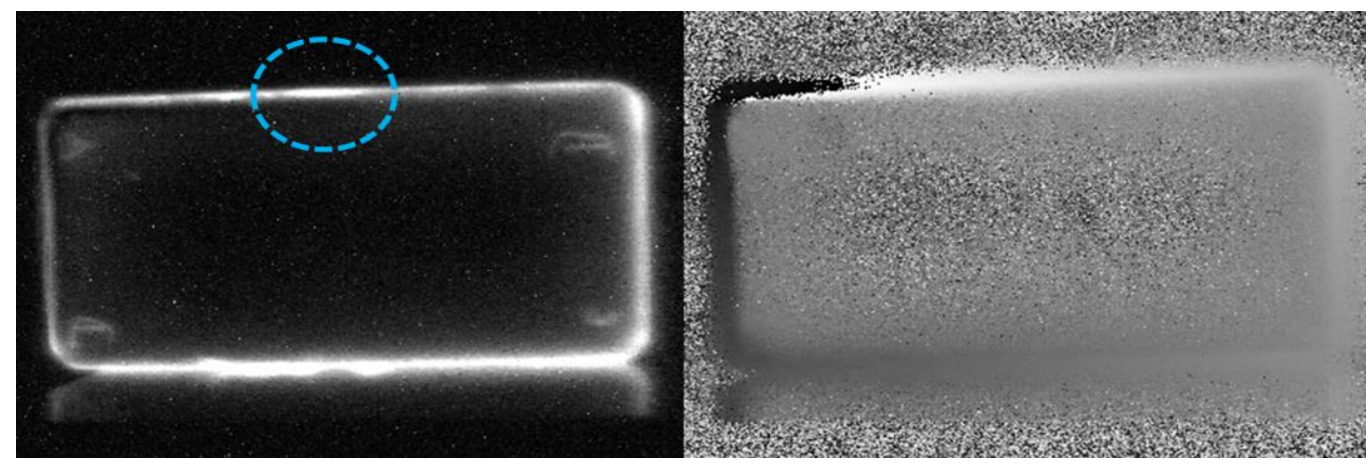

Fig. 4. Thermal amplitude image (left) and phase image (right) of the sample. The phase scale ranges from $-180^{\circ}$ (black) to $+180^{\circ}$ (white)

As expected, the amplitude image is non-zero only in the heat affected zone around the laser beam path. Local inhomogeneities can be detected. At the marked, but also at other positions there are inhomogeneities visible which are mainly due to slight surface corrosion of the steel sheet. The phase image in Fig. 4 is dominated by the geometrical phase term. In the upper left part of the path one can see the phase jump from $-180^{\circ}$ to $+180^{\circ}$.

A second measurement is performed with inverse rotational direction. The total time needed for both measurements is $30 \mathrm{~s}$. Then, the averaged phase image is calculated according to eq. (6). The result is shown in Fig. 5. In order to suppress phase noise far from the measurement path, the phase was set zero at pixels where the corresponding amplitude falls below a certain threshold.

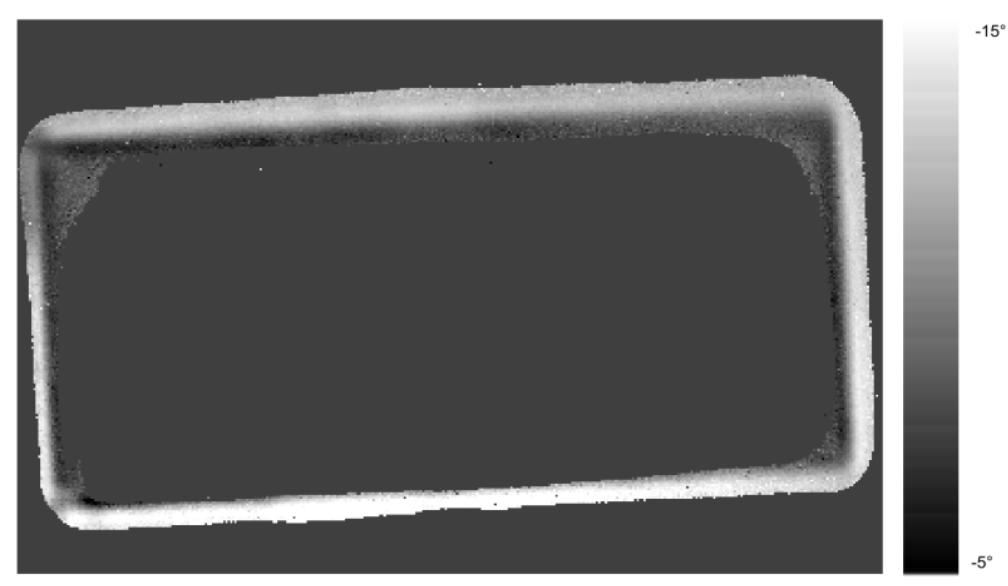

Fig. 5. Flying-spot lock-in phase image of the metal sheet sample

Now, local changes of the phase can be seen at the curved edges, which are due to thickness variations. In order to process the phase contrast further, an analytical solution of the thermal wave transmission through the sheet was used. The transmitted temperature oscillation $\theta$ through a metal sheet of thickness $d$ is given by

$$
\Theta=\frac{Q_{0}}{\lambda_{s} \sigma_{s}} \frac{2}{(1+g)^{2} e^{\sigma_{s} d}-(1-g)^{2} e^{-\sigma_{s} d}} \text {, with } \sigma_{\mathrm{s}}=(1+\mathrm{i}) /\left(\alpha_{\mathrm{s}} /\left(\pi \mathrm{f}_{\mathrm{m}}\right)\right)^{1 / 2} \text {, }
$$


where $Q_{0}$ is the absorbed energy density, $\lambda_{s}$ the thermal conductivity of the sheet, $\alpha_{s}$ its thermal diffusivity and $g$ the effusivity ratio between air and the sheet $(g<<1)$.

This results in a calibration curve for the modulation frequency of $1.56 \mathrm{~Hz}$ as shown in Fig. 6 .

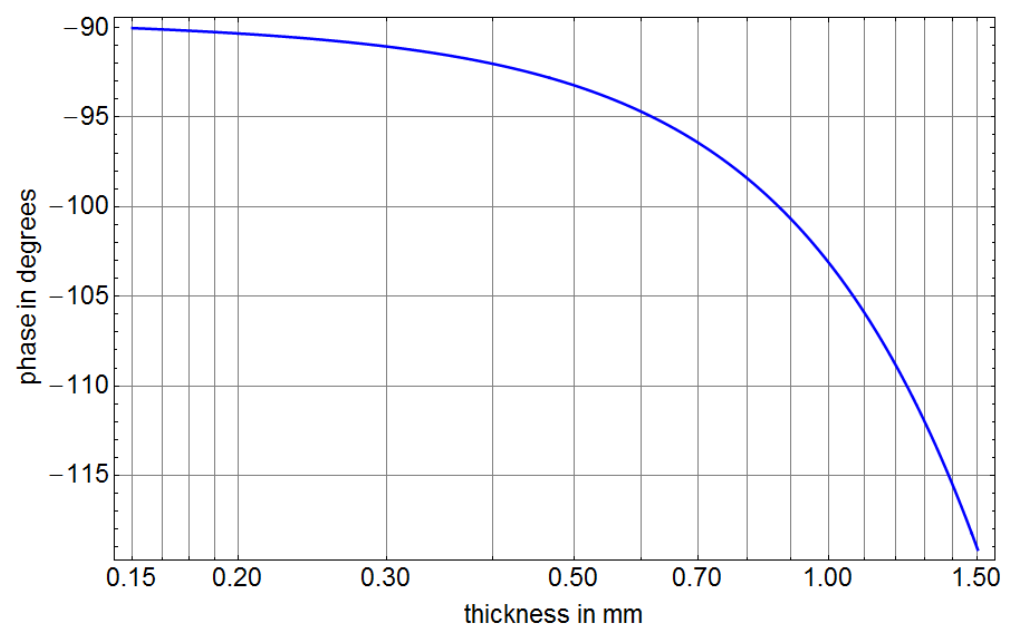

Fig. 6. Calculated calibration curve between sheet thickness and thermal phase for a modulation frequency of $1.56 \mathrm{~Hz}$

This curve still has to be adapted to the measured phase signal at a reference point by using a constant phase offset, which represents the unknown fixed phase shifts in the measurement system. The sheet thickness at the reference point was determined by a high-frequency ultrasound time-of-flight measurement. Finally, the calibration curve from Fig. 6 is used to generate the map of sheet thickness at the edge shown in Fig. 7. According to this, at the lower edge the sheet thickness is slightly lower as at the upper edge. In the sample corners, local thickness variations can be seen at the left side. The measured thickness variation from the ultrasound measurements was somewhat smaller as the one shown in Fig 7. Exact verification by ultrasound turned out to be difficult due to the surface curvature.

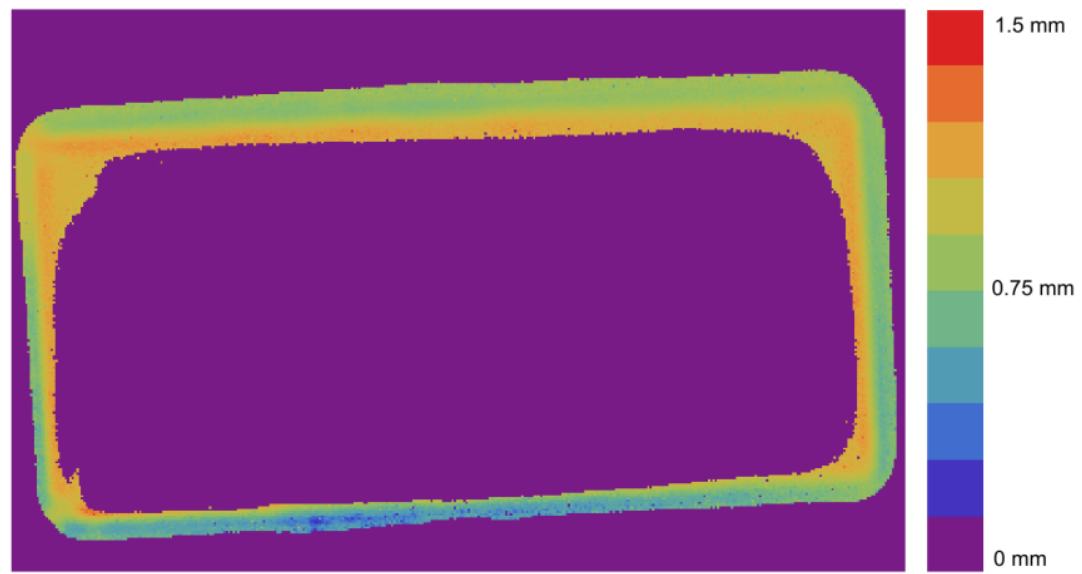

Fig. 7. Image of the sheet thickness at the sample edges calculated from the phase image (Fig. 5) using the calculated calibration function

\section{Crack detection}

The flying-spot lock-in technique was also demonstrated for the detection of a perpendicular surface crack. A steel sample containing a machined crack of $200 \mu \mathrm{m}$ width and about $20 \mathrm{~mm}$ length open to the surface was scanned along a rectangular laser path as shown in Fig. 8, left. The sample was blackened in order to increase the emissivity. A measurement in thermal reflection was performed in a similar way as described before. The laser spot with a power of $10.7 \mathrm{~W}$ was moved at a speed of $10.2 \mathrm{~mm} / \mathrm{s}$. Ten cycles around the path were recorded. The modulation frequency was $0.1 \mathrm{~Hz}$. The lock-in procedure was performed as described above, resulting in the amplitude and phase images shown in Fig. 8, centre and right. 

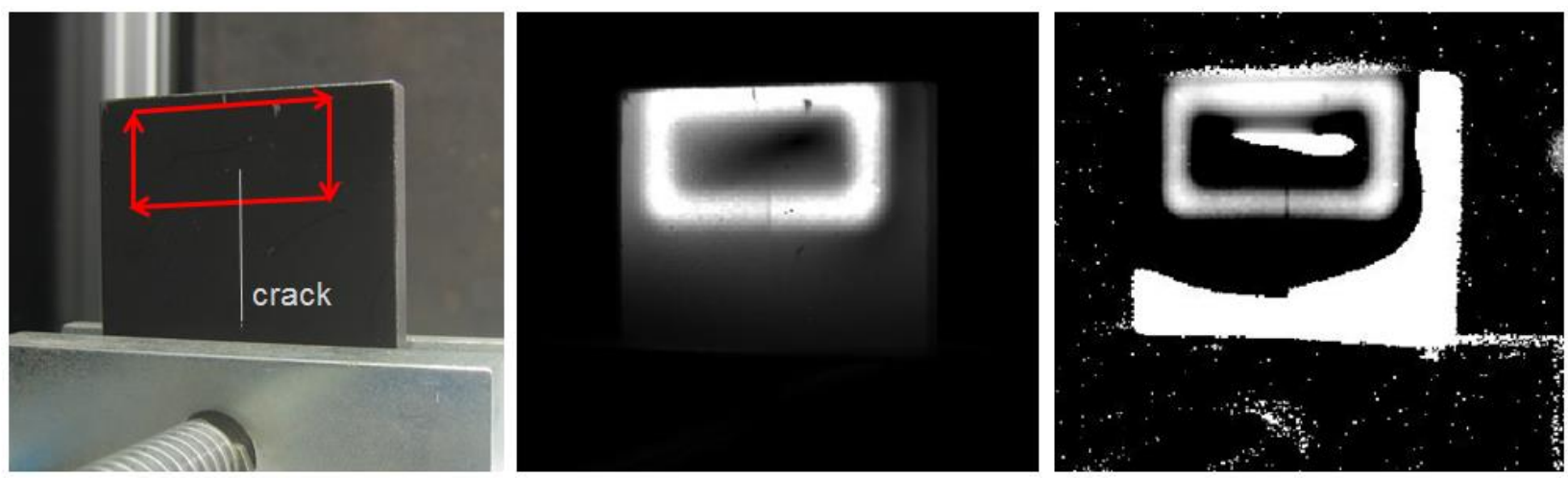

Fig. 8. Left: Sample photograph with laser path marked in red and the position of the crack marked in white. Centre: amplitude image. Right: averaged phase image

The results show that the crack can be detected in the amplitude and in the phase images. In Fig. 9, line profiles of the amplitude and phase along the path over the crack are shown. The amplitude profiles for the clockwise (CW) and counter-clockwise (CCW) beam movement reveal the typical asymmetry around the crack due to the blocking of the heat flow parallel to the surface. The phase profiles show the geometrical phase shift and a phase jump at the crack. After averaging, a local phase minimum remains at the crack position. Figs. 8 and 9 also show that emissivity effects in the amplitude due to damage in the black coating are well suppressed in the phase signal.

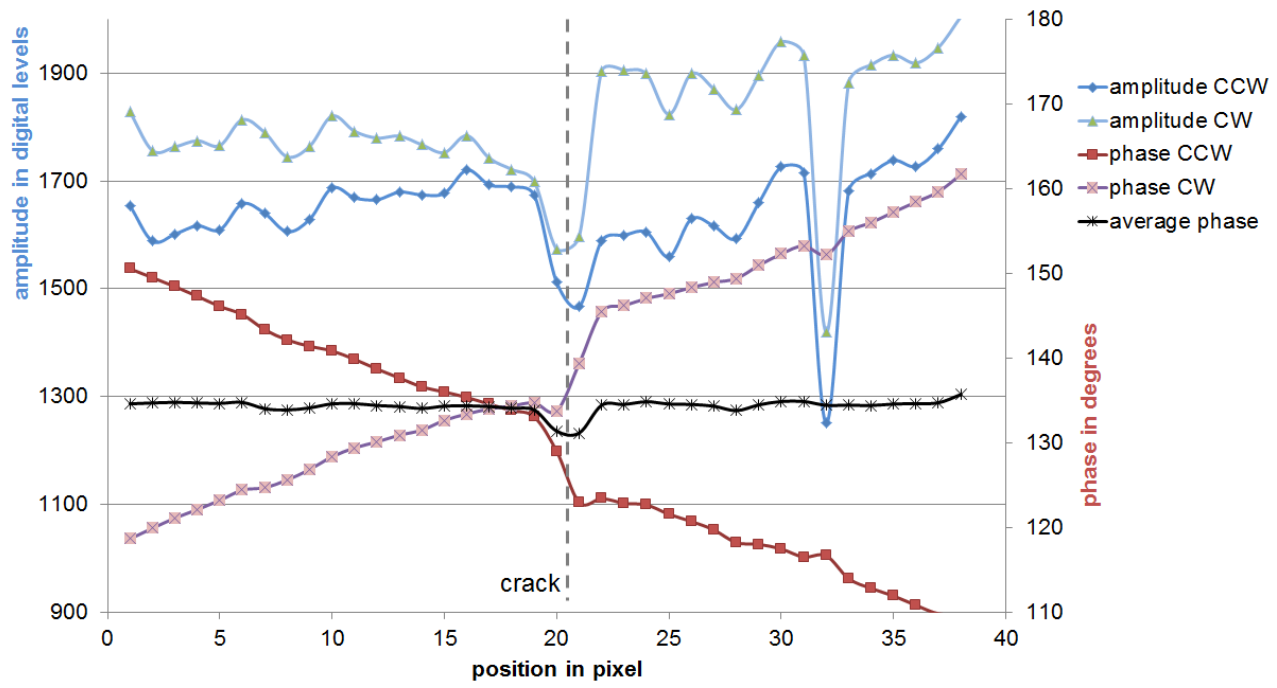

Fig. 9. Profiles of amplitude and phase along a line over the crack (line length about $8 \mathrm{~mm}$ ). The profiles are shown for counter-clockwise (CCW) and clockwise (CW) movement of the laser spot

\section{Discussion}

Some approximations and open questions concerning flying-spot lock-in thermography should be addressed. There is a deviation from the classic lock-in technique insofar as periodic pulses instead of sinusoidal signal are used for excitation. This may lead to additional phase shifts, as higher harmonics will also generate phase contributions. However, they are negligible, if a usual sin/cos correlation algorithm with at least 10 frames per modulation period is used to calculate amplitude and phase.

Laser beam movement also generates phase effects, similar to photothermal experiments on moving objects. [15]. This remains to be investigated. Due to thermal diffusion in the direction perpendicular to the laser path, phase shifts are occurring that may be misinterpreted as thickness variations. In a thermally isotropic material, the beam diameter should therefore be large compared to the layer or sheet thickness.

The summation of two phase images with opposite sense of beam rotation leaves the signal amplitude unconsidered. Vector summation may be the better proceeding. 


\section{Conclusion}

Using a periodic, comparably fast cw-laser beam movement along a testing path, amplitude and phase images can be generated along the beam path using suitable data processing. Available lock-in algorithms for active thermography can readily be used with a simple post-processing. In the application on formed steel components, disturbances were suppressed efficiently and a plot of the steel thickness distribution was generated within $30 \mathrm{~s}$ measurement time. The technique may help to reveal surface cracks with better contrast.

Flying-spot lock-in will be of interest, where not a full surface, but only a limited area prone to failure has to be tested, e. g. the region along weld lines. For full surface inspection, the approach could be extended to periodic laser line scanning.

\section{REFERENCES}

[1] E. Kubiak, 'Infrared detection of fatigue cracks and other near-surface defects', Appl. Optics 7 (1968) 1743

[2] I. Kaufman, P. T. Chang, H. S. Hsu, W. Y. Huang, and D. Y. Shyong, 'Photothermal radiometric detection and imaging of surface cracks', J. Nondestr. Eval. 6 (1987) 87

[3] J. Hartikainen, J. Jaarinen and M. Luukkala, 'Delamination and crack detection by the synchronous heating method: theoretical aspects', Rev. Progr. QNDE 8B, (1989) 1321

[4] Y. Q. Wang, P. K. Kuo, L. D. Favro, and R. L. Thomas, 'Flying laser spot thermal wave IR imaging of horizontal and vertical cracks', Rev. Progr. QNDE 9, (1990) 511

[5] C. Gruss, F. Lepoutre, and D. Balageas, 'Non destructive evaluation using a flying spot camera', Proc. 6th ECNDT, Nice 1994, p. 657

[6] T. Li, D. Almond, D. Rees, 'Crack imaging by scanning laser-line and laser-spot thermography', Meas. Sci. and Technology 22 (2011) 035701

[7] F. Lepoutre and D. Balageas, 'Non destructive evaluation using a flying spot camera', Proc. 6th ECNDT, Nice (1994) 657

[8] J.-C. Krapez, L. Legrandjacques, F. Lepoutre, and D. Balageas, 'Optimization of the photothermal camera for crack detection', QIRT open archives, Proc. 4th QIRT, Lodz, Poland, 1998, paper 48

[9] M. Ziegler, M. Kreutzbruck, C. Maierhofer, M. Pelkner, J. Schlichting, 'Kameragestütztes Flying-Spot-Verfahren zur Risserkennung', Thermographie-Kolloquium 2011, Vortrag 12, DGZfP-Berichtsband 130-CD (Deutsche Gesellschaft für zerstörungsfreie Prüfung (DGZfP, Berlin) 2011

[10] U. Netzelmann and H. Zhang, 'Prüfung von Keramikoberflächen mit Wärmewellenmikroskopie', DGZfP Berichtsband 38, Jahrestagung 9.-11.5.1994, Timmendorfer Strand, (DGZfP, Berlin 1995) 745-751

[11] H. Petry, 'Online-Messung von Lackschichten mit thermischen Wellen', tm 65, 396 (1998)

[12] G. Busse, 'Thermal-wave experiments on moving samples', Can. J. Phys. 64, (1986) 1281

[13] I. A. Sazonov, 'Propagation of Harmonic Thermal Waves in a Shear Flow', Acoustical Physics 40, 735 (1994)

[14] U. Netzelmann, 'Analysis of thermal wave propagation in coatings on moving objects', Progress in Natural Science (China) 6, (1996) 235

[15] C. Gruss, R. Hüttner, B. Bein, 'Theoretical and experimental analysis of a photothermal modulation technique for application on moving samples', Proc. 13th Int. Conf. on Thermal Engineering and Thermogrammetry, Budapest 2003 